\title{
Analytical Approach for Channel Assignments in Cellular Networks ${ }^{\star}$
}

\author{
Vladimir V. Shakhov ${ }^{1}$ and Hyunseung Choo $^{2}$ \\ 1 Institute of Computational Mathematics and Mathematical Geophysics, \\ Siberian Branch of the Russian Academy of Sciences, \\ 630090, Novosibirsk, Russia \\ shakhov@rav.sscc.ru \\ 2 School of Information and Communication Engineering \\ Sungkyunkwan University, Suwon 440-746, Korea \\ choo@ece.skku.ac.kr
}

\begin{abstract}
In the present paper channel assignments in cellular architectures are considered. This is quite important in cell and channel planning since reusing channels in cells allows to manage resources and to serve users effectively in the system. The optimal solution is offered here for the case of co-channel interference. Previous solutions on co-channel interference are based on regular hexagonal models for service areas. A novel technique is employed in this work that does not depend upon any geometric form of cells. It is obtained that the optimal number of channels equals the density of a special graph. Earlier analytical results on span minimization show lower bounds meanwhile we provide the upper bound in this paper.
\end{abstract}

\section{Introduction}

A mobile computing has become an essential part of modern telecommunication. As demands for wireless mobile communication grow under limited resources of cellular systems, it is very important to use frequency channels as efficiently as possible to maintain the necessary quality of services. Thus, frequency reuse is the key concept of the cellular network design [1]. According to the concept the same frequency channel can be simultaneously used in different cells. The geographical distance between cells should be sufficiently large. Otherwise, interference may decrease the quality of service. So, the goal of Channel Assignment Problem is an effective utilization of frequency region under some interference constraints.

There exist two types of interference constraints. 1) The co-channel constraint, where the same channel cannot be assigned to certain pairs of radio cells simultaneously. An interference between different channels is absent for any

\footnotetext{
* This work was supported in part by grant No. 2000-2-30300-004-3 from the Basic Research Program of Korea Science and Engineering Foundation. Dr. H.Choo is the corresponding author.
} 
cells. Transmitters having a mutual distance no smaller than admissible distance $r$ may use the same frequencies, when $r$ is a known constant. 2) The adjacent channel constraint, where any couple of assigned channels must be separated by a certain frequency band. This band depends on the physical distance between cells which use the channels simultaneously. If the distance is sufficiently large then the band equals zero.

The interference constraints generate two kinds of Channel Assignment Problem. Let us consider the restriction 1). We have some quantity of potential channels. Each cell should get a set of available frequency channels under constraint 1). It is necessary to minimize a number of used channels in the cellular system. We name it as minimization of the number of channels (MNC). The next kind of Channel Assignment Problem is generated by restriction 2). Let us remark that the constraint 2) generalizes the constraint 1). Here we have a bandwidth. Frequency channels are extracted from the bandwidth and assigned to cells by taking into account the constraint 2). It is required to minimize the bandwidth which is used by the cellular system. In literature the technical term span is applied [4]. The span of an assignment is the difference between the largest and the smallest channels used [12. Therefore, it needs to find the minimum span over all possible assignments. We name this as Span Minimization (SM).

For channel assignments a simplified model of cellular network is used. A service area of cellular network has been modeled by a net of regular homogeneous hexagonal cells 451781012. It is a good approximation for the use of omnidirectional antennas. Let us remark the hexagon is non-unique model of a cell. A cell can be a triangle if antennas of cellular network have a sector direction [2]. If an office network is designed, rectangular cells are used [3]. A geometry of cells can be used by methods for frequency assignment.

Now we shall give the following definition. The cellular graph is a graph where each cell of the cellular network is represented by a node and two nodes have a common edge between them if the corresponding cells are adjacent to each other [7. Frequency assignment problems are reduced to a problem of cellular graph coloring. For an arbitrary graph the problem is NP complete. However, the optimal number of channels is found by using some properties of cellular graphs. Without loss of generality it is assumed that a set of available frequencies for a cell consists of a single element 48].

In previous works MNC under regular hexagonal cell system was investigated and an optimal solution has been found. For this system the optimal number of frequencies is given in 6]. In [4] MNC has been considered for Euclidean admissible distance $r$ and a channel assignment algorithm has been offered. The case that $r$ equals a number of cells was named $r$-distance problem and investigated in [7,8]. However, results discussed are out-of-use for other cell systems. A cell can be non-hexagonal, $r$ can be different for other part of service area and so on. In this paper we present an alternative technique for computing the the optimal number of channels, which can be more widely applicable.

Generally, SM is an NP-complete problem [5/13]. The optimal solution of the problem is obtained only for particular cases [14]. In [15] a branch and bound 
algorithm is used, but this approach does not guarantee against the complete enumeration. For a large-scale system an approach for the optimal solution is impractical. Thus a simplification of the problem or an approximation technique have been used, such as neural network based algorithms [161718, simulations 1920, and genetic algorithms 921|22 23. Many authors have studied lower bounds for SM. The most popular bounds are based on cliques 78 or on minimum weight Hamiltonian paths 1112. The technique for mathematical programming has been applied for lower bounds [12,5]. In this paper we offer unimprovable upper bound for SM.

The paper is organized as follows. In Section 2, MNC is considered. We prove the optimal number of channel is defined by the density of special graph. In Section 3, SM is investigated. An upper bound for frequencies assignment under is offered with some examples. Section 4 is a brief conclusion.

\section{On Minimizing the Number of Channels}

Let us consider a service area covered by omnidirectional base stations. Admissible distance $r$ can be differ for other part of service area. Let $G(V, E)$ be a cellular graph, when $V$ is the vertex set and $E$ is the edge set. By $d(u, v)$ denote distance from $u$ to $v$, when $u, v \in V$. Now we shall give the following definitions.

Definition 1. A graph is called $r$-graph and is denoted by $G_{r}$ if $V\left(G_{r}\right)=$ $V(G), E\left(G_{r}\right)=\{(u, v): d(u, v) \leq r$ in $G\}$

Definition 2. A complete subgraph of $G_{r}$ is a cluster if it is not contained by other complete subgraphs.

It is clear MCN is reduced to the graph coloring problem for $G_{r}$. The optimal number of channels for the regular hexagonal cell system equals a power of cluster 6 $6,4,7,8$. Here we do not use any assumption for geometric form of cells.

Lemma 1. Let the service area be completly covered by cells, i.e. there will be no non-signalling (empty) zone. Then the corresponding cellular graph is chordal.

Proof. Without loss of generality we may consider a simple cycle with 4 nodes in cellular graph. The service area of four corresponding base stations has no empty zone. Let two non-adjacent vertices of the cycle have no edge between them. The cell areas for those two base stations are non-overlapping sets. Let $P$ be the service area within the cycle excluding those two cell areas. Denote by $A$ and $B$ service zones of other base stations into $P$. It is clear that $P \cap A \neq \emptyset$ and $P \cap B \neq \emptyset$. It follows from the lemma condition that $P=A \cup B$. We have $P \backslash A \neq \emptyset$. Hence, $P \backslash A \subset B$ or $P \backslash A=B$, i.e. set $B$ is the complement of set $A$. So, if two vertices is non-adjacent then other vertices should be adjacent. The proof is completed. $\diamond$

Let us remark $E\left(G_{r}\right) \supset E(G)$. Hence $G_{r}$ is a chordal graph too. The following theorem is needed for the sequel.

Theorem 1. Every chordal graph is perfect [24]. 
It is known that the chromatic number of the perfect graph is equal to the graph density. So, we have

Theorem 2. The optimal number of channels equals the density of corresponding r-graph.

\section{Upper Bound for Span Minimization}

Here we use the same notation as in the section above. By $f_{i}$ denote a frequency assigned to cell $i$. An admissible frequency assignment will be a set of positive numbers $\left\{f_{i}\right\}$ such that

$$
\left|f_{i}-f_{j}\right| \geq c_{i, j} \quad \forall i, j \in V .
$$

The lowest frequency equals 0 . Hence, span $F$ is the maximum frequency assigned to the system. That is,

$$
F=\max f_{i}, \quad i \in V \text {. }
$$

It is necessary to find min $F$ among all admissible assignments. A compatibility matrix is a symmetric matrix $C=\left(c_{i, j}\right)$ with nonnegative integer entries $c_{i, j}$ [5. We say that $C$ is the distance compatibility matrix if the following conditions hold

$$
\forall i, j, u, v \in V: c_{i, j}=c_{u, v} \text { if } d(i, j)=d(u, v) .
$$

As in the literature, the distance compatibility matrix is assumed and let us have values $s_{0}, s_{1}, s_{2}, \ldots$, such that

$$
c_{i, i}=s_{0} \forall i \in V \text { and } \forall i, j \in V: c(i, j)=s_{k} \text { if } d(i, j)=k, k \in\{1 \ldots n-1\} .
$$

It is clear that $s_{0} \geq s_{1} \geq s_{2} \ldots$

As in 8.9. we consider the problem for a single mobile user in each cell. This assignment can be used for more number of users. Suppose $F_{1}$ is the assignment for a single customer per cell, the frequency $f_{i}$ is assigned to cell $i$, and we have $K$ calls per cell. If $s_{0}$ is sufficiently large, then the assignment $f_{i}, f_{i}+$ $s_{0}, \ldots f_{i}+(K-1) s_{o}$ is used in cell $i$, else we use the assignment $f_{i}, f_{i}+F_{1}+$ $s_{0}, \ldots f_{i}+F_{1}+(K-1) s_{o}$. Generally, it is not admissible for an optimal solution nor lower bound. However, it is acceptable for upper estimation. Let us consider the following example (See Figure 1). We have cellular network $(A, B, C, D)$ and one customer in each cell. Interference constraints are $s_{0}=s_{1}=s$. The optimal span equals $2 s$ and the distribution of frequencies is shown. We receive the same results. However, if two customers are served in cell $D$, we receive span $4 s$ using our approach, meanwhile the optimal span equals $2 s$. Let us remark if we have $K$ customers in each cell (homogeneous traffic) then an optimal solution is reached by the approach above.

Now we offer the following technique for the frequency assignment of one user per cell which produces the unimprovable upper bound of a span and an 


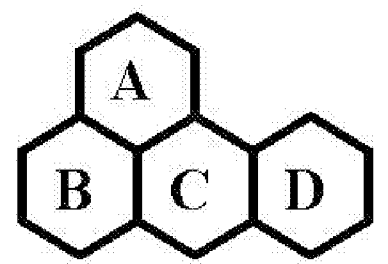

Cellular network

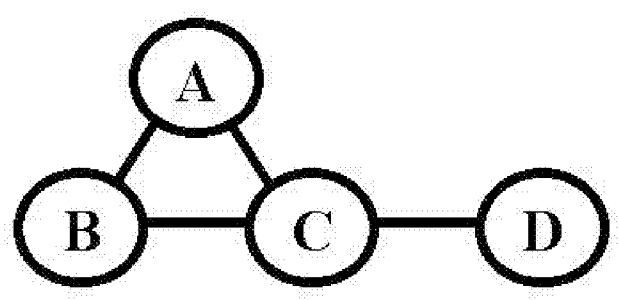

Cellular graph

\begin{tabular}{|l|c|c|c|c|c|c|c|c|}
\hline cells & A & B & C & D \\
\hline $\begin{array}{l}\text { number of } \\
\text { customers }\end{array}$ & 1 & 1 & 1 & 1 \\
\hline $\begin{array}{l}\text { our } \\
\text { assignment }\end{array}$ & $\mathrm{s}$ & $2 \mathrm{~s}$ & 0 & $\mathrm{~s}$ \\
\hline $\begin{array}{l}\text { optimal } \\
\text { assignment }\end{array}$ & $\mathrm{s}$ & $\mathbf{2 s}$ & 0 & $\mathrm{~s}$ \\
\hline
\end{tabular}

Fig. 1. The example of frequency assignment

admissible frequency assignment. For some particular cases this assignment will be an optimal.

1) Let $G(V, E)$ be a cellular graph. We consider only interference constrain $s_{1}$ and decide the graph coloring problem for $G$. By $\chi$ denote the chromatic number of $G$, i.e. colors $0,1, \ldots \chi-1$ are used. If the node $i$ received color $k$, then $f_{i}=k s_{1}$. In adjacent cells $i, j$ we have $\left|f_{i}-f_{j}\right| \geq s_{1}$. If $s_{2}=0$ then $\mathrm{SM}$ is decided and $F=(\chi-1) s_{1}$.

2) Let $s_{2} \neq 0$. Suppose the vertices $i, j$ have the same color. If $d(i, j)=2$, then corresponding vertices cannot use the same channel. The set $V$ is divided in subsets $V_{i}, i=1, \ldots, \chi$, where elements of $V_{i}$ have the same color in $G$. Now we decide the graph coloring problems for graphs $G_{i}, i=1, \ldots \chi$, where $V\left(G_{i}\right)=V_{i}$ and an edge $(u, v) \in E\left(G_{i}\right)$ if $d(u, v)=2$ in $G$. Let the chromatic number of $G_{1}$ be $\chi_{i}$. If the node $i \in G_{1}$ received color $k$, then $f_{i}=k s_{2}$. In adjacent cells $i, j$ of $G_{1}$ we have $\left|f_{i}-f_{j}\right| \geq s_{2}$ but the condition for $s_{1}$ should be true too, i.e. $\left|\max f_{i}-\min f_{j}\right|=s_{1}, i \in G_{1}, j \in G_{2}$ Hence, for nodes of $G_{1}$ we use frequencies $0, s_{2}, \ldots,\left(\chi_{1}-1\right) s_{2}$, for nodes of $G_{2}$ we use frequencies $\left(\chi_{1}-1\right) s_{2}+s_{1}, \chi_{1} s_{2}+s_{1}, \ldots,\left(\chi_{1}+\chi_{2}-2\right) s_{2}+s_{1}$, and so on. If $s_{3}=0$ then $\mathrm{SM}$ is completed and $S=\left(\chi_{1}+\chi_{2}+\ldots+\chi_{\chi}-\chi\right) s_{2}+(\chi-1) s_{1}$. If $s_{3} \neq 0$, then the process above is repeated, and so on, until $s_{j}=0, j=4,5 \ldots$.

An example for $s_{3} \neq 0$ is on figure 2 (3-band buffering system). It is clear the offered method get upper bound for SM. Let us consider example from [8] (see figure 3 ). Here $s_{1}>s_{2} \neq 0, s_{3}=0$. The assigned frequency channels are into 

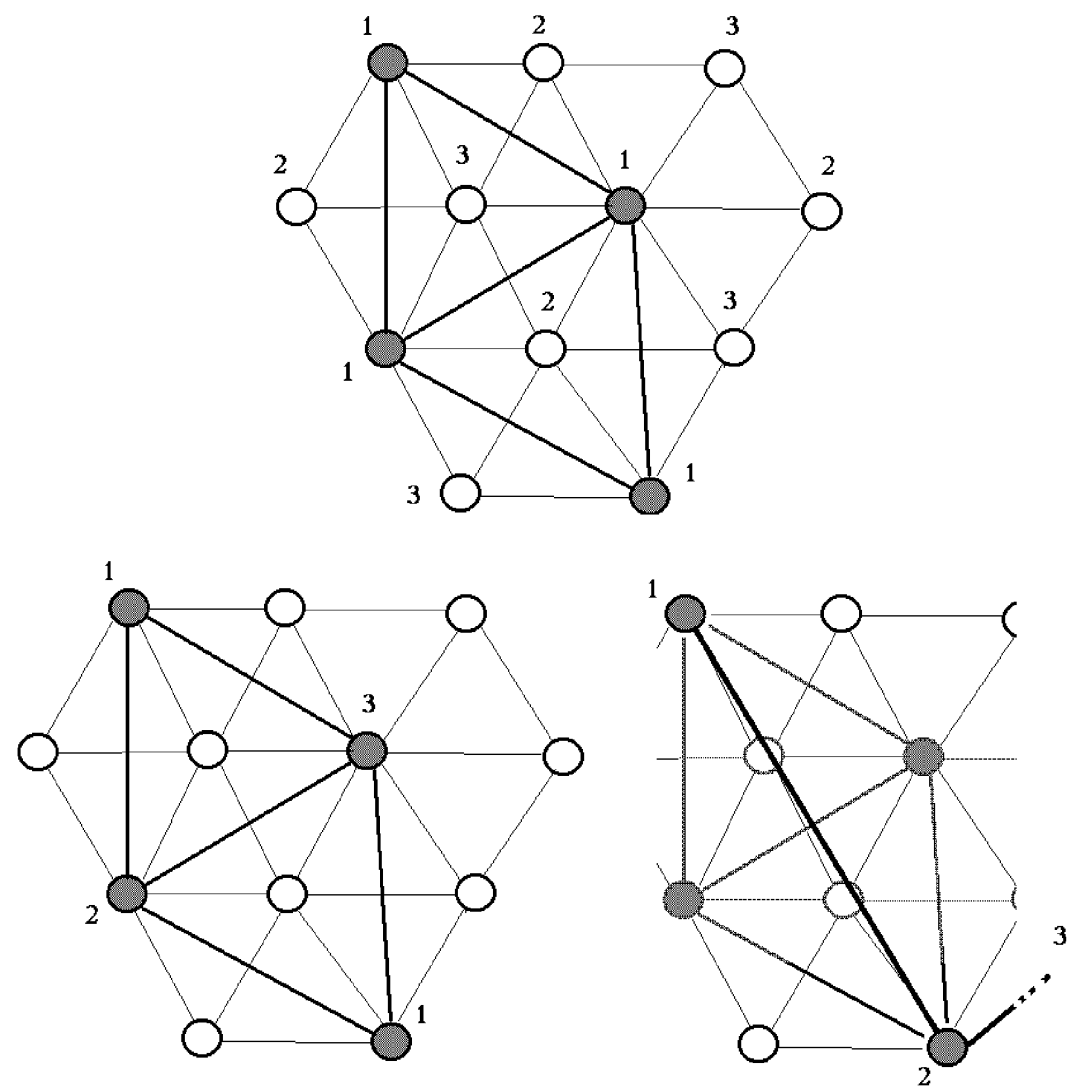

Fig. 2. 3-band buffering system.

vertices. A couple $(\mathrm{a}, \mathrm{b})$ in node mean that corresponding cell receive channel $a s_{1}+b s_{2}$. Our method gives the same assignment. If $s_{1}<2 s_{2}$, then the shown solution is optimal,hence, we received the unimprovable upper bound. Else, the solution can be improved as it shown on the figure.

\section{Conclusion}

In most of real cellular networks, the homogeneous hexagonal model is unrealistic. We have obtained the optimal number of channels under co-channel interference for more practical cellular networks which are not necessary homogeneous and hexagonal. It is proved that the optimal number of channels equals the density of r-graph constructed from cellular graphs. For the span minimization the upper bound is obtained. The division of compatibility matrix is used and sequential solution of graph coloring problems are made. The distance between 


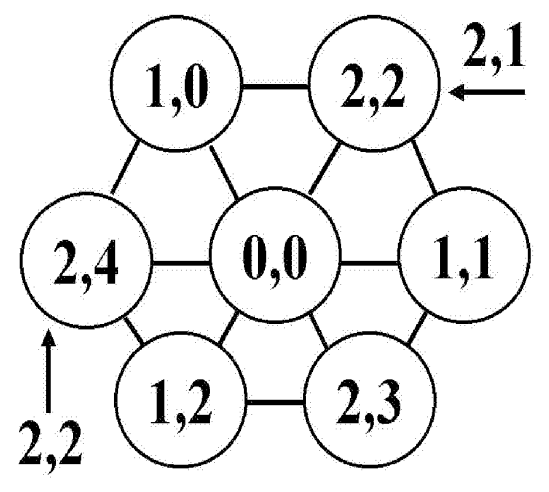

Fig. 3. Example of solution

cells is defined as a path in cellular graphs and we consider the distance compatibility matrix. Our approach is applicable to Euclidean distance and an arbitrary compatibility matrix.

\section{References}

1. W. Lee, Mobile Cellular Telecommunications: analog and digital systems, Second Edition, New York: McGraw-Hill, 1995.

2. Y. Akaiwa, Intoduction to Digital Mobile Communications, New York: John Wiley \& Sons, 1997.

3. S. Fedortsov, B. Tsybakov, "Channels distribution in cellular network," Information Transmition Problems, vol. 32, pp. 91-99, 1996 (in Russian).

4. A. Gamst, "Homogeneouse Distribition of Frequencies in a Regular Hexaginal Cell System," IEEE Transactions on Veh. Technology, vol. VT-31, pp. 132-144, Aug. 1982.

5. A. Gamst, "Some Lower Bounds for a Class of Frequency Assignment Problems," IEEE Transactions on Veh. Technology, vol. VT-31, pp. 132-144, Aug. 1982.

6. V.H. MacDonald, "Advanced mobile phone service: The cellular concept," Bell Syst. Tech. J., v.58, pp. 15-41, 1979.

7. A. Sen, T. Roxoborough, and S. Medidi, "Upper and lower Bounds of a Class of Channel Assignment Problems in Cellular Networks," Proc. of IEEE INFOCOM'98, vol. 3, pp. 1284-1291, 1998.

8. A. Sen, T. Roxoborough, and S. Medidi, "On an Optimal Algorithm for Channel Assignment in Celluar Networks," Proc. of IEEE ICC'99, vol. 2, pp. 1147-1151, 1999.

9. C.Y. Ngo and V.O.K. Li, "Fixed channel assignment in cellular radio networks using a modified genetic algorithm," IEEE Transactions on Vehicular Technology, v.47, No.1, pp. 163-172, 1998.

10. J.A. Khan, S.M. Sait, and S.A. Khan, "A fast constructive algorithm for fixed channel assignment problem," The 2001 IEEE International Symposium on Circuits and Systems, v.5, pp. 65-68, 2001. 
11. C.W. Sung and W.S. Wong, "Sequential Packing Algorithm for Channel Assignment Under Cochannel and Adjacent Channel Interference Constraint," IEEE Transactions on Vehicular Technology, v.46, No.3, pp. 676-686, 1997.

12. D.H. Smith, S. Hurley, and M. Allen, "A new lower bound for the channel assignment problem," IEEE Trans. Veh. Technol., v.49, No.4, pp. 1265-1272, 2000.

13. W.K. Hale, "Frequency assignment: Theory and application," Proc. IEEE, v.68, pp. $1497-1514$.

14. J.C.M. Janssen and K. Kilakos, "An Optimal Solution to the "Philadelphia" Channel Assignment Problem," IEEE Trans. Veh. Technol., v.48, No.3, pp. 1012-1014, 1999.

15. S.Z. Ali and L.F. Turner, "An efficient methodology for optimal channel assignment of large and complex mobile radio networks," IEEE Trans. Veh. Technol., VTC 2001 Fall. IEEE VTS 54th, v.1, pp. 389-393, 2001.

16. D. Kunz, "Channel assignment for cellular radio using neural network," IEEE Trans. Veh. Technol., v.40, No.1, pp. 188-193, 1991.

17. N. Funabiki and Y.Takefuji, "A neural network parallel algorithm for channel assignment problems in cellular radio network," IEEE Trans. Veh. Technol., v.41, pp. 430-437, 1992.

18. N.A. El-Fishawy, M.M. Hadhood, S. Elnoubi, and W. El-Sersy, "A modified Hopfield neural network algorithm for cellular radio channel assignment," IEEE VTS Fall VTC 2000. 52nd, v.3, pp. 1128-1133, 2000.

19. M. Duque-Anton, D. Kunz, and B. Ruber, "Channel assignment for cellular radio using simulation annealing," IEEE Trans. Veh. Technol., v.42, pp. 14-21, 1993.

20. R. Mathar and J. Mattfeldt, "Channel assignment in cellular radio networks," IEEE Trans. Veh. Technol., v.42, pp. 647-656, 1993.

21. W.K. Lai and G.G. Coghill, "Channel assignment through evolutionary optimization," IEEE Trans. Veh. Technol., v.45, No.1, pp. 91-96, 1996.

22. K.A. Smith, "A genetic algorithm for the channel assignment problem," IEEE Global Telecommunications Conference, v.4, pp. 2013-2018, 1998.

23. G. Chakraborty and B. Chakraborty "A genetic algorithm approach to solve channel assignment problem in cellular radio networks," Proc. of SMCia/99, pp. 34-39, 1999.

24. R. Diestel, Graph Theory, Second Edition. Springer-Verlag, New York, pp. 111$112,2000$. 